\title{
Rotational motion of a stationary axisymmetric relativistic heat conducting fluid configuration
}

\begin{abstract}
The present work describes some dynamical features associated with the dissipation caused by the heat flow in the context of rotational motion of a heat conducting fluid based on Carter's two-fluid model. A covariant solutions to a pair of Maxwell's like equations governing the evolution of a heat conducting fluid are used to determine the heat flow vector and the matter part of fluid's 4-velocity under the assumption that the space-time configuration representing the gravitational field of a heat conducting fluid is non-circular stationary and axisymmetric. These results are used to derive the rotational velocity in terms of gradients of the effective energy and effective angular momentum per particle. It is demonstrated that the meridional circulations of the matter part of fluid induce the rotational velocity in addition to the usual rotational velocity found in a circular space-time. It is shown that the differential rotation along the thermal -fluid vorticity causes the twist of matter part of fluid's vortex lines in addition to the other effects. It is found that the gravitational isorotation is balanced by counter rotating thermal isorotation in the case of differentially rotating thermal-fluid surfaces in a frame of reference in which thermal -fluid helicity is conserved. The dynamic interaction between the matter part of fluid and the entropy fluid leads to the mutual exchange between the energy and angular momentum per baryon of the matter part of fluid and the flux of energy and angular momentum per entropon per unit of local temperature coupled to heat flow vector created by the entropy fluid.
\end{abstract}

Keywords: thermal-fluid vorticity, thermal-fluid helicity, heat conducting fluid, thermal isorotation
Volume 2 Issue 6 - 2018

\author{
G Prasad \\ Retired Principal, Kamla Nehru Institute of Physical and Social \\ Sciences, Dr. R. M. L. University, India
}

\begin{abstract}
Correspondence: G Prasad, Kamla Nehru Institute of Physical and Social Sciences, affiliated to Dr. R . M. L. University, Faizabad (U.P.), India, Email gumrajpr@gmail.com
\end{abstract}

Received: October 07, 2018 | Published: November 20, 2018

\section{Introduction}

A qualitative understanding of thermal dynamics is of considerable interest in order to investigate the underlying physical processes during the evolution of newly born neutron stars which are far from thermodynamic equilibrium because such stars at birth are sensitive to high temperature variations. ${ }^{1}$ The structural aspects and thermal evolution of such compact stars require determination of background space-time geometry and the equation of state (EOS) of highly degenerate hot nuclear matter that composes the stars. The dissipative processes that occur involve on the one hand strong gravitational effects and on the other hand microscopic properties of dense matter exceeding nuclear densities. The behavior and composition of hot matter at large densities are still unknown. Nevertheless, the usual working hypothesis is that the hot degenerate matter can be thought of as a heat conducting fluid in a hydrodynamic approximation.

In construction of a theoretical model of a differentially rotating neutron star, it is usually assumed the background space-time geometry representing the gravitational field is stationary and axisymmetric. ${ }^{2}$ The condition of thermodynamic equilibrium asserts that the entropy current must be divergence- free. ${ }^{3}$ This condition leads to the vanishing of heat flow and Born rigidity ${ }^{4}$ of fluid flow lines. ${ }^{3}$ In this case fluid composing the star attains barotropic EOS predicted on the basis of Eckart's theory of dissipative fluids ${ }^{5}$ because of the argument that the dissipative effects due to heat flow and viscosity die out. ${ }^{3}$ In this case , it is found that twist of fluid's vortex lines vanishes. ${ }^{6}$

Since a newly born neutron star is in a state of differential rotation, the flows of hot fluid composing such star will not obey Born rigidity condition ${ }^{4}$ because the shear tensor associated with fluid flow lines is non-vanishing due to the star's differential rotation. ${ }^{8,9}$ The presence of heat flow due to inhomogeneous high temperature distribution and differential rotation will keep the star far from thermodynamic equilibrium. As a result, the energy flux generated due to heat flow and differential rotation leads to the twisting of fluid's vortex lines. ${ }^{10}$ This in turn suggests that the role of fluid's vorticity cannot be ignored when a hot fluid is far from thermodynamic equilibrium. It is also important to underline that the fluid's vorticity is a basic element when coupled to fluid's chemical potential forms the fluid's helicity. ${ }^{11}$

Some recent theoretical and numerical investigations ${ }^{12-13}$ indicate that the study of thermal evolution of a newly born neutron star is important for understanding physical processes of observed thermal radiation from such stars on the basis of cooling theory. ${ }^{14}$ But the way the energy balance equation is formulated ${ }^{12-13}$ for the study of rotational effects on thermal evolution of a newly born neutron star violates the causality principle because the formulation involves Fourier's law for the description of heat conduction and an analogous construction of energy-momentum tensor based on Eckart's theory of dissipative fluids. ${ }^{5}$ It is well known that Fourier's law causes the causality breakdown. This in turn suggests that the predictions made in the context of thermal evolution and undergoing physical processes due to the interaction between the motion of a hot fluid and the heat flow inside a newly born neutron star on the basis of such formulation may not be physically reasonable on the ground of causality violation. Effort to better formulation for the description of thermal evolution in the case of a rotating star is still ongoing. ${ }^{15}$

The space-time configuration representing the gravitational field of a self-gravitating heat conducting fluid in a state of differential rotation is unknown because of the absence of exact analytic solutions 
to Einstein field equations incorporating causal theory of heat conducting fluid. In the case of Carter's two-fluid mode ${ }^{16}$ of a heat conducting fluid, it is recognized that the heat flow vector contributes to an additional thermal stress term coupled with the entropy entrainment ${ }^{17}$ that enters in the expression of energy-momentum tensor but does not appear in Israel and Stewart (I S) causal theory ${ }^{18}$ of a dissipative fluid. This additional thermal stress term is linked with gravitational potentials via components of metric tensor described by Einstein field equations. It is worthwhile to note that Carter's model ${ }^{16}$ is not only causal and stable but also includes IS theory ${ }^{18}$ as a special case. ${ }^{19}$ The entrainment between matter part of fluid and the entropy fluid is the key ingredient for incorporating thermal relaxation time that leads to causal heat transport in a modified version of Carter's two-fluid model. ${ }^{17}$ The dynamical features associated with the motion of such two-fluid model are unknown and deserve to be investigated in view of its applicability to a system composed of heat conducting fluid which is far from thermal equilibrium. Because of the existence of non-conserved physical quantities like thermal-fluid vorticity flux, thermal vorticty flux, thermal-fluid helicity, thermal helicity etc. ${ }^{10,20}$ describing dissipation, there is extreme difficulty encountered in finding analytic solutions to Einstein field equations. This in turn suggests that the search of some conserved physical quantity of interest might be helpful to extract useful information in order to understand the physical processes associated with the dynamics of dissipative fluid.

The central elements of Carter's variational model ${ }^{16}$ are the existence of two distinct vorticity 2-forms corresponding to the matter part of fluid and the entropy fluid and are called, respectively, the particle vorticity 2 -form and the thermal vorticity 2 -form. These two distinct vorticity 2 -forms comprise a pair of two distinct sourcefree Maxwell's like equations ${ }^{16}$ that describe the evolution of a heat conducting fluid. This pair of equations exhibits that there is an intrinsic interdependence of matter part of fluid's 4-velocity and the heat flow. If the space-time configuration representing the gravitational field of a heat conducting fluid is assumed to be axisymmetric and stationary, it must be non-circular because the energy-momentum tensor does not obey the circularity condition ${ }^{21}$ and the presence of meridional circulation of matter part of fluid is mandatory ${ }^{20}$ for the survival of covariant toroidal components of the heat flow vector in a modified version of Carter's model. ${ }^{17}$ Furthermore, the vanishing of covariant toroidal components of the heat flow vector exclude the contributions to the effective energy and the effective angular momentum per particle that results in the reduction of the EOS to a function of state variables represented by particle number density and the constant entropy per baryon describable by the usual Gibbs law; while this is contrary to the fact that the EOS of a heat conducting fluid in this model is a function of three thermodynamic state variables , i.e., the particle number density, the entropy density and their entrainment factor governed by the extended Gibbs law. ${ }^{17}$ This means that the non- circularity assumption is essential if the space-time is assumed to be stationary and axisymmetric. But the non-circularity assumption in the case of an axisymmetric and stationary space-time leads to the appearance of non-diagonal metric tensor components in Einstein field equations that make the solution intractable. Since the evolution of a heat conducting fluid is governed by a pair of source-free Maxwell's like equations, an electromagnetic analogy based on the approach given ${ }^{22}$ for the case of the relativistic magneto hydrodynamics (RMHD) ${ }^{23}$ can be employed to seek similar solutions to the case of a heat conducting fluid. On the basis of this approach, ${ }^{22}$ it is found that there is conservation of thermal chirality associated with the non-conserved thermal helicity ${ }^{20}$ if the entropy fluid flows are axisymmetric stationary without meridional circulation but with uniform rotation. Thus it seems appropriate to continue this approach for further study of dynamic features associated with the interplay between the motion of matter part of fluid and the entropy fluid.

The present work is focused to extend our understanding of rotational motion of a system composed of a heat conducting fluid based on Carter's two-fluid model. ${ }^{16,17}$ The paper is organized as follows. Section. 2 is devoted to a brief summary of relevant results ${ }^{20}$ that are required for our present work. Section. 3 is concerned with the derivation of a relation connecting the differential rotation of matter part of fluid and the thermal-fluid vorticity under the assumption that the space-time is axisymmetric stationary and non-circular. Section. 4 is intended to describe the differential rotation of thermal-fluid vorticity flux surfaces in a frame of reference in which thermal-fluid helicity is conserved and to derive a Ferraro's like law of isorotation. We deduce a relation between gravitational isorotation and thermal isorotation. Section.5 is devoted to the derivation of conservation laws of the energy and angular momentum currents associated with the motion of a heat conducting fluid by invoking space-time symmetry assumption. We show that there is mutual exchange between the energy per baryon of matter part of fluid and the flux of effective energy per entropon per unit of local temperature coupled to heat flow vector generated by the entropy fluid in the dynamic interaction. Similar result will be shown for the case of the angular momentum.

Convention: The space-time metric is of signature +2 .Small case Latin indices run from 0 to 3 . Semicolon and comma are used, respectively, to denote the covariant and partial derivatives. Constituent indices $n$ and $s$ are used to indicate matter and entropy part of fluid, respectively, throughout the text and not to be confused with tensor indices. Capital Latin indices are used to indicate poloidal coordinates. Square and round brackets around indices represent, respectively, skew-symmetrization and symmetrization.

\section{Covariant Solutions to Maxwell's like equations in stationary sxisymmetric space- time}

In this section, we collect all relevant results which are required for the present work. These results can be found in $^{10,20}$ but are briefly stated here for the convenience of reader. The starting point is a brief summary of Catrer's variational model ${ }^{16,17}$ of a heat conducting fluid in which the thermal-fluid vorticity 2 -form $W_{a b}$ and thermal vorticity 2-form $Z_{a b}$ constitute a pair of source free Maxwell's like equations that govern the evolution of a heat conducting fluid. In this model, a heat conducting fluid is composed of matter current $n^{a}$ and entropy current $s^{a}$. The entropy current element is thought of as a massless fluid (e.g., phonons/entropons). In order to preserve causality in the matter frame of reference (also called Eckart's frame), ${ }^{5}$ the notion of thermal relaxation time is incorporated via the formulation of a relativistic version of Cattaneo's equation by invoking the entropy entrainment ${ }^{17}$. It is found that Eckart's frame ${ }^{5}$ is not only suitable to connect the traditionally known thermodynamic variables expressing the equation of state (EOS) of a fluid but also to take accounts of the kinematic deformation of fluid flow and heat flow lines caused by gravitation via the curvature of space-time. A pair of Maxwell's like equations describing the evolution of a heat conducting fluid are expressible as: ${ }^{10,16,17}$

$$
\begin{aligned}
& W_{a b} u^{b}=\mathrm{E}_{a} \\
& Z_{a b} s^{b}=-\mathrm{E}_{a}
\end{aligned}
$$


with

$$
\begin{aligned}
& W_{a b}=2 \mu_{[b ; a]}, Z_{a b}=2 \vartheta_{[b ; a]} \\
& \mathrm{E}_{a}=\frac{R}{n}\left({ }^{*} s-\frac{\beta q^{2}}{2}\right) q_{a} \quad \overline{\mathrm{E}}_{a}=R\left(q_{a}-\frac{q^{2}}{{ }^{*} \theta} u_{a}\right)
\end{aligned}
$$

where $\mu_{a}={ }^{*} \mu_{u_{a}}+\alpha q_{a}$ is the fluid momentum convector and $\vartheta_{a}={ }^{*} \theta u_{a}+\beta q_{a}$ is the thermal momentum convector. The fluid's 4-velocity is denoted by $u^{a}$ which obeys the normalization condition $u^{a} u_{a}=-1$. The matter current takes the form $n^{a}=n u^{a}$ which is conserved, that is, $n_{; a}^{a}=0$. This is usually called the baryon conservation law. $q^{a}$ is the heat flow vector. The entropy current is represented by $s^{a}={ }^{*} S u^{a}+\frac{1}{{ }^{*} \theta} q^{a}$. The fluid's chemical potential, temperature, and entropy per baryon measured in the matter part of fluid's frame are, respectively, designated by ${ }^{*} \mu,{ }^{*} \theta$ and ${ }^{*} s$. The relation between thermodynamic quantities $\alpha$ and $\beta$ is expressible as $\beta=\left(\frac{1}{{ }^{*}}-\frac{n \alpha}{{ }^{*}{ }_{s}}\right)$, where $\alpha=\frac{A^{n s}}{{ }^{*} \theta}$ which encodes the entrainment effect via $A^{n s} \cdot{ }^{17}$ The thermal resistivity of a thermally conducting fluid is described by $R$. The electric part of the thermal -fluid vorticity 2-form $W_{a b}$ is represented by $E_{a}$. Its magnetic part is defined by

$$
V^{a}={ }^{*} W^{a b} u_{b}
$$

Where ${ }^{*} W^{a b}$ denotes the Hodge dual of $W^{a b}$ and is defined by ${ }^{*} W^{a b}=\frac{1}{2} \eta^{a b c d} W_{c d}$. Here $\eta^{a b c d}$ is the Levi-Civita skew symmetric tensor. The magnetic part $V^{a}$ is expressible as: $:^{10}$

$$
V^{a}=2\left({ }^{*} \mu \omega^{a}+\alpha q \hat{\omega}^{a}\right)
$$

where $\omega^{a}=\frac{1}{2} \eta^{a b c d} u_{b} u_{c ; d}=\frac{1}{2} \eta^{a b c d} u_{b} \omega_{c d}$ is the matter part of fluid's vorticity tensor. ${ }^{24} \omega_{c d}$ is the rotation tensor associated with the timelike congruence of matter part of fluid's flow lines. The space like twist of heat flow lines is denoted by $\hat{\omega}^{a}$ and can be defined by invoking Greenberg's theory ${ }^{25}$ of spacelike congruence as $\hat{\omega}^{a}=\frac{1}{2} \eta^{a b c d} m_{b} \hat{\omega}_{c d}$, where $m^{a}$ is the unit spacelike vector field directed along the heat flow vector $q^{a}$ and obeys the normalization condition $m^{a} m_{a}=1$. The rotation tensor associated with the spacelike congruence of heat flow lines is represented by $\hat{\omega}_{a b}$ and is defined by $\hat{\omega}_{a b}=p_{a}^{c} p_{b}^{d} m_{[c ; d]}$, where $p_{b}^{a}=\delta_{b}^{a}+u^{a} u_{b}-m^{a} m_{b}$ is the projection tensor orthogonal to both $u^{a}$ and $m^{a}$. The construction of the magnetic part $V^{a}$ is subjected to the following condition

$$
\stackrel{\circ}{m}_{a}=(\ln \alpha q)_{, a}
$$

where $\dot{m}_{a}=m_{a ; b} m^{b}$ is the curvature vector of heat flow lines. ${ }^{10}$ The magnetic part $V^{a}$ is referred to as thermal -fluid vorticity vector. ${ }^{10}$

From now on, we assume that the space-time representing the gravitational field of a heat conducting fluid is axisymmetric stationary and non-circular. This means that there is a pair of two linearly independent Killing vectors of which one is time like Killing vector $\xi_{(t)}^{a}$ generating a translational symmetry with open timelike lines as orbits and the other one is a spacelike Killing vector $\xi_{(\varphi)}^{a}$ generating rotations about a symmetry axis. ${ }^{21}$ There exists a family of invariant timelike 2-surfaces, called surfaces of transitivity, generated by this pair of Killing vectors that correspond to ignorable coordinates $x^{0}=t$ and $x^{3}=\varphi$ (i.e. $\xi_{(t)}^{a}=\delta_{t}^{a}$ and $\left.\xi_{(\varphi)}^{a}=\delta_{\varphi}^{a}\right) .{ }^{21}$ The ignorable coordinates $t$ and $\varphi$ are called toroidal coordinates. This pair of Killing vectors constitutes the basis of tangent plane tangential to surface of transitivity. Its dual basis can be constructed as follows: ${ }^{22}$

$$
\begin{aligned}
& { }^{*} \xi_{(t)}^{a}=\frac{1}{K}\left(-g_{\varphi \varphi} \xi_{(t)}^{a}+g_{t \varphi} \xi_{(\varphi)}^{a}\right) \\
& { }^{*} \xi_{(\varphi)}^{a}=\frac{1}{K}\left(g_{t \varphi} \xi_{(t)}^{a}-g_{t t} \xi_{(\varphi)}^{a}\right)
\end{aligned}
$$

with the properties

$$
{ }^{*} \xi_{(t)}^{a} \xi_{(t) a}=1={ }^{*} \xi_{(\varphi)}^{a} \xi_{(\varphi) a} \text { and }{ }^{*} \xi_{(t)}^{a} \xi_{(\varphi) a}=0^{*} \xi_{(\varphi)}^{a} \xi_{(t) a}
$$

where

$$
K=g_{t \varphi}^{2}-g_{t t} g_{\varphi \varphi}>0
$$

The thermal -fluid vorticity 2 -form $W_{a b}$ is constituted by matter particle canonical momentum convector given by $\mu_{a}=u_{a}+\alpha q_{a}$. The matter part of fluid's 4-velocity $\mu_{a}$ can be decomposed into toroidal and poloidal components as follows: ${ }^{22}$

$$
u^{a}=\zeta\left(\xi_{(t)}^{a}+\Omega \xi_{(\varphi)}^{a}\right)+w^{a},
$$

where $w^{a}$ denotes meridional circulation velocity of the matter part of fluid and is orthogonal to both $\xi_{(t)}^{a}$ and $\xi_{(\varphi)}^{a}$. The quantity $\zeta=\sqrt{\frac{1+w^{2}}{B}}$, where $B=-\left(g_{t t}+2 \Omega g_{t \varphi}+\Omega^{2} g_{\varphi \varphi}\right)$. Since the matter part of thermal- fluid vorticity 2-form $W_{a b}$ must respect the symmetry assumption, we have

$$
£_{\xi_{(t)}} W_{a b}=0=£_{\xi_{(\varphi)}} W_{a b} .
$$

where $£_{\xi_{(t)}}$ and $£_{\xi_{(\varphi)}}$ denote, respectively, the Lie derivative with respect to $\xi_{(t)}^{a}$ and $\xi_{(\varphi)}^{a}$.

Using (2.7) in the first relation of (2.1c), we find that

$$
W_{a b} \xi_{(t)}^{b}=\bar{\varepsilon}_{, a} \text { and } W_{a b} \xi_{(\varphi)}^{b}=\bar{j}_{, a},
$$

where $\mu_{t}={ }^{*} \mu_{u_{t}+\alpha q_{t}}=\bar{\varepsilon}_{, a}$ and $\mu_{t}={ }^{*} \mu_{u_{\varphi}+\alpha q_{\varphi}}=\bar{j} \quad$ denote, respectively, the effective energy and the effective angular momentum per particle corresponding to the matter part of fluid. ${ }^{26}$ From (2.8), the thermal-fluid vorticity 2-form $W_{a b}$ and its Hodge dual ${ }^{*} W^{a b}$ are obtainable in the following form:

$$
W_{a b}=2 \bar{\varepsilon}_{,\left[a^{*} \xi(t) b\right]}-2 \bar{j}_{,\left[a^{*} \xi(\varphi) b\right]}+\frac{\bar{I}}{K} \eta_{a b c d} \xi_{(t)}^{c} \xi_{(\varphi)}^{d}
$$

and

$$
{ }^{*} W^{a b}=\eta^{a b c d} \bar{\varepsilon},{ }_{c}^{*} \xi_{(t) d}-\eta^{a b c d}{ }_{c}^{*} \xi_{(\varphi) d}-\frac{\bar{I}}{K}\left(\xi_{(t)}^{a} \xi_{(\varphi)}^{b}-\xi_{(\varphi)}^{a} \xi_{(t)}^{b}\right)
$$

where $\bar{I}={ }^{*} W_{a b} \xi_{(t)}^{a} \xi_{(\varphi)}^{b}$.

Contraction of (2.9) with $u^{b}$ with the aid of (2.3) and the first relation of $(2.1 \mathrm{~d})$, reduces (2.1a) to yield that 
$\mathrm{E}_{a}=\zeta\left(\bar{\varepsilon}_{, a}-\Omega \bar{j}_{, a}\right)-\left(\bar{\varepsilon}_{, b} w^{b}\right)^{*} \xi_{(t) a}+\left(\bar{j}_{, b} w^{b}\right)^{*} \xi_{(\varphi) a}+\frac{\bar{I}}{K} \eta_{a b c d} w^{b} \xi_{(t)}^{c} \xi_{(\varphi)}^{d}$

Setting $C=\frac{1}{n}\left(-\frac{\beta q^{2}}{2}\right)$ in the first relation of (2.1d), we obtain from (2.11) that

$$
q_{a} \oplus \frac{1}{R C} \zeta\left\lfloor\left(\bar{\varepsilon}_{, a}-\Omega \bar{j}_{j, a}\right)-\left(\bar{\varepsilon}_{, b} w^{b}\right)^{*} \xi_{(t) a}+\left(\bar{j}_{, b} w^{b}\right)^{*} \xi_{(\varphi) a}+\frac{\bar{I}}{K} \eta_{a b c d} w^{b} \xi_{(t)}^{c} \xi_{(\varphi)}^{d}\right\rfloor,
$$

which determines the heat flow vector field in terms of the gradients of effective energy per particle and effective angular momentum per particle and the meridional circulation velocity of the matter part of fluid. Making use of (2.5a) and (2.5b) in (2.12), one may obtain

$$
q_{a}=\frac{1}{R C}\left\lfloor\zeta\left(\bar{\varepsilon}_{, a}-\Omega \bar{j}_{, a}\right)+\frac{A_{1}}{K} \xi_{(t) a}-\frac{A_{2}}{K} \xi_{(\varphi) a}+\frac{\bar{I}}{K} \eta_{a b c d} w^{b} \xi_{(t)}^{c} \xi_{(\varphi)}^{d}\right\rfloor,
$$

where

$$
A_{1}=\left(\bar{\varepsilon}_{, a} w^{a}\right) g_{\varphi \varphi}+\left(\bar{j}_{, a} w^{a}\right) g_{t \varphi} \text { and } A_{2}=\left(\bar{\varepsilon}_{, a} w^{a}\right) g_{t \varphi}+\left(\bar{j}_{, a} w^{a}\right) g_{t t} \text {. }
$$

An explicit expression for thermal-fluid vorticity vector is obtainable as follows. Contracting (2.10) with $u_{a}$ and making use of (2.6) and $(2.5 \mathrm{a}, \mathrm{b})$ in the resulting expression, we find that

$$
V^{a}=\frac{1}{K}\left[-\bar{I}_{\varphi} \xi_{(t)}^{a}+u_{t} \xi_{(\varphi)}^{a}-\eta^{a b c d} w_{b}\left(Q_{c} \xi_{(t) d}-\bar{Q}_{c} \xi_{(\varphi) d}\right)-u_{t} \eta^{a b c d} R_{b} \xi_{(t) c} \xi_{(\varphi) d}\right],
$$

where

$$
\begin{aligned}
& Q_{c}=g_{\varphi \varphi} \bar{\varepsilon}_{, c}+g_{t \varphi} \bar{j}_{, c}, \bar{Q}_{c}=g_{t \varphi} \bar{\varepsilon}_{, c}+g_{t t} \bar{j}_{, c} \text { and } R_{b}=\frac{u_{t}}{\zeta}\left(\bar{j}_{, b}-l \bar{\varepsilon}_{, b}\right), \\
& l=-\frac{u_{\varphi}}{u_{t}}, u_{t}=\zeta\left(g_{t t}+\Omega g_{t \varphi}\right), \text { and } u_{\varphi}=\zeta\left(g_{t \varphi}+\Omega g_{\varphi \varphi}\right) .
\end{aligned}
$$

In order to determine the matter part of fluid's 4-velocity $u^{a}$, we use (2.1b) which describes the evolution of the entropy fluid. Setting $s^{a}=s u_{s}^{a}$, where $u_{s}^{a}=\gamma\left(u^{a}+v_{s}^{a}\right)$ which satisfies $u_{a} v_{s}^{a}=0$. The red

$$
u_{a}=\frac{{ }^{*} S^{*} \theta}{R q^{2}}\left[\left(\frac{\zeta}{C}\right)\left(\bar{\varepsilon}_{, a}-\Omega \bar{j}_{, a}\right)+\left(\frac{\lambda}{\gamma}\right)\left(\varepsilon_{, a}-\Omega_{s} j_{, a}\right)-B_{1}{ }^{*} \xi_{(t) a}+B_{2}{ }^{*} \xi_{(\varphi) a}+\frac{1}{K} \eta_{a b c d}\left(\frac{\bar{I}}{C} w^{b}+\frac{I}{\gamma} v^{b}\right) \xi_{(t)}^{c} \xi_{(\varphi)}^{d}\right]
$$

where

$$
B_{1}=\frac{1}{C}\left(\bar{\varepsilon}_{, b} w^{b}\right)+\frac{1}{\gamma}\left(\varepsilon_{, b} v^{b}\right) \text { and } B_{2}=\frac{1}{C}\left(\bar{j}_{, b} w^{b}\right)+\frac{1}{\gamma}\left(j_{, b} v^{b}\right) \text {. }
$$

It is evident from (2.25) that the matter part of fluid's 4-velocity depends on gradients of effective energy and effective angular momentum per particle corresponding to both the matter part of fluid and the entropy fluid and is coupled to the meridional circulations of both fluids. Eq. (2.25) underlines the significance of dynamic interaction of the motion of both fluids. Contracting (2.25) with $\xi_{(t)}^{a}$ and $\xi_{(\varphi)}^{a}$, in turn, we find that

$$
u_{t}=-B_{1} \text { and } u_{\varphi}=B_{2} \text {, }
$$

shift factor is $\gamma=\left(1-v_{s}^{2}\right)^{-\frac{1}{2}}$ and ${ }^{*} s=\gamma s$. With this substitution, we transform (2.1b) in the following form

$$
Z_{a b} u_{s}^{b}=-{ }_{a},
$$

where

$$
\tilde{\mathrm{E}}_{a}=\gamma R\left(q_{a}-\frac{q^{2}}{{ }^{*} S^{*} \theta} u_{a}\right) \text {. }
$$

We now decompose thermal vorticity 2-form $Z_{a b}$ by invoking the space-time symmetry assumption. Because of symmetry assumption thermal vorticity 2 -form $Z_{a b}$ obeys the following conditions

$$
£_{\xi_{(t)}} Z_{a b}=0=£_{\xi_{(\varphi)}} Z_{a b},
$$

Since $Z_{a b}$ is a curl, we obtain from (2.19) that

$$
Z_{a b} \xi_{(t)}^{b}=\varepsilon_{, a} \text { and } Z_{a b} \xi_{(\varphi)}^{b}=-j_{, a} \text {, }
$$

where

$$
\vartheta_{t}={ }^{*} \theta u_{t}+\beta q_{t}=-\varepsilon \text { and } \vartheta_{\varphi}={ }^{*} \theta u_{\varphi}+\beta q_{\varphi}=j \text {. }
$$

Here $\varepsilon$ and $j$ denote, respectively, effective thermal energy and effective thermal angular momentum per particle like entropon (as is named by $\mathrm{Carter}^{26}$ ) in the sense of Carter. ${ }^{26}$ Using (2.20) we can express thermal vorticity 2 - form as follows:

$$
Z_{a b}=2 \varepsilon_{,[a}{ }^{*} \xi_{(t) b]}-2 j_{,[a}{ }^{*} \xi_{(\varphi) b]}+\frac{I}{K} \eta_{a b c d} \xi_{(t)}^{c} \xi_{(\varphi)}^{d},
$$

where $I={ }^{*} Z_{a b} \xi_{(t)}^{a} \xi_{(\varphi)}^{b}$. The entropy fluid's 4-velocity $u_{s}^{a}$ can be decomposed into toroidal and poloidal components given by

$$
u_{s}^{a}=\lambda\left(\xi_{(t)}^{a}+\Omega_{s} \xi_{(\varphi)}^{a}\right)+v^{a} .
$$

where $v^{a}$ represents the meridional velocity of motion of the entropy fluid and $\lambda=\sqrt{\frac{1+v^{2}}{A}}$, and $A=-\left(g_{t t}+2 \Omega_{s} g_{t \varphi}+\Omega_{s}^{2} g_{\varphi \varphi}\right)$.

Contracting (2.22) with $u_{s}^{b}$ with the aid of (2.23), we find that

$$
-\tilde{\mathrm{E}}_{a}=\lambda\left(\varepsilon_{, a}-\Omega_{s} j_{, a}\right)-\left(\varepsilon_{, b} v^{b}\right)^{*} \xi_{(t) a}+\left(j_{, b} v^{b}\right)^{*} \xi_{(\varphi) a}+\frac{I}{K} \eta_{a b c d} v^{b} \xi_{(t)}^{c} \xi_{(\varphi)}^{d} .
$$

It follows from (2.12), (2.18) and (2.24) that

which, in view of (2.26), says that covariant toroidal components of matter part of fluid's 4-velocity survive even if its meridional circulation vanishes because of non-vanishing meridional circulation of the entropy fluid . But it can be seen from (2.13) and (2.14) that $q_{t}=0=q_{\varphi}$ if the meridional circulation velocity of the matter part of fluid is zero. This result when used in the relation below (2.8) and (2.21) we see that the contributions due to toroidal components of heat flow vector couple with the entropy entrainment to the effective energy per particle and the effective angular momentum per particle of both fluids are excluded. This in turn implies that the zero meridional circulation of the matter part of fluid is contrary to the basic fact that EOS of a heat conducting fluid is a function of three thermodynamic state variables, namely that, the baryon number density, entropy 
density, and the entropy entrainment factor. Thus we arrive at the conclusion that the existence of matter part of fluid's meridional circulation is mandatory in a Carter's model ${ }^{16,17}$ of a heat conducting fluid. However, the zero meridional circulation of the entropy fluid does not lead to such contradiction. With these remarks, we proceed to investigate the rotational motion of the matter part of fluid.

\section{Rotational motion of matter part of fluid}

This section is concerned with the description of rotational velocity of the matter part of fluid because the rotational velocity represented by $\Omega$ in a circular space-time will be modified due to the contribution of the presence of meridional circulations in a non-circular spacetime. The rotational velocity in a non-circular axisymmetric stationary space-time with the aid of decomposition of matter part of fluid's 4-velocity given by (2.6) is obtainable as

$$
\bar{\Omega}=\frac{u^{\varphi}}{u^{t}}=\frac{\zeta \Omega+w^{\varphi}}{\zeta+w^{t}}
$$

which can be linearized in the following form

$$
\bar{\Omega} \approx \Omega+\overline{\bar{\Omega}},
$$

where

$$
\overline{\bar{\Omega}}=\frac{1}{\zeta}\left(w^{\varphi}-\Omega w^{t}\right) .
$$

In consequence of ignoring the second and higher order terms in the expansion.It is obvious from (3.3) that $\overline{\bar{\Omega}}$ represent an additional rotation induced by the meridional circulation of the matter part of fluid to that of the usual rotation $\Omega$ found in circular space-time. Substituting $w^{\varphi}=-\frac{1}{K}\left(g_{t \varphi} g_{t A}-g_{t t} g_{\varphi A}\right) u^{A}$ and $w^{t}=\frac{1}{K}\left(g_{\varphi \varphi} g_{t A}-g_{t \varphi} g_{\varphi A}\right) u^{A}$ (see for details $\left.{ }^{27}\right)$ in (3.3) and making use of $g_{t \varphi}=-g_{\varphi \varphi}$, we get

$$
\overline{\bar{\Omega}}=\frac{1}{\zeta K}\left[\left(g_{t t}-\varpi \Omega g_{\varphi \varphi}\right) g_{\varphi A} u^{A}-g_{\varphi \varphi}(\Omega-\varpi) g_{t A} u^{A}\right]
$$

Where $\varpi$ represents the frame dragging effect ${ }^{28}$ which contributes to the additional rotation $\overline{\bar{\Omega}}$ caused by meridional circulation of the matter part of fluid.

On account of second and third relations of (2.16), we find from (2.27) after some calculations that

$$
\Omega=-\frac{1}{\zeta K}\left(C^{-1} D_{1}+\gamma^{-1} D_{2}\right)
$$

where

$$
D_{1}=g_{t \varphi}\left(\bar{\varepsilon}_{, a} w^{a}\right)+g_{t t}\left(\bar{j}_{, a} w^{a}\right) \text { and } D_{2}=g_{t \varphi}\left(\varepsilon_{, a} v^{a}\right)+g_{t t}\left(j_{, a} v^{a}\right) \text {. }
$$

From (3.5) and the second relation of (3.6), we observe that the meridional circulation velocity of the entropy fluid also contributes to the rotational velocity of the matter part of fluid.

Following Bardeen, ${ }^{28}$ we define the injection energy per particle corresponding to both the matter part of fluid and the entropy fluid, respectively, as follows

$\bar{\Phi}=\bar{\varepsilon}-\Omega \bar{j}$ and $\Phi=\varepsilon-\Omega_{s} j$

which yields that

$$
\varepsilon_{, a}-\Omega_{s} j_{, a}=\Phi_{, a}+j \Omega_{s, a}
$$

From (2.13) and the first relation of (3.8), we find that

$$
w^{A}=\frac{1}{I} €^{A B}\left[K R C q_{B}-K \zeta\left(\bar{\Phi}_{, B}+\bar{j} \Omega_{, B}\right)-A_{1} g_{t B}+A_{2} g_{\varphi B}\right],
$$

which gives the meridional circulation velocity of the matter part of fluid. The alternating symbol $€^{A B}$ takes values 1 or -1 for poloidal indices. It follows from (3.4) and (3.9) after some calculations that

$$
\overline{\bar{\Omega}}=\frac{1}{I \zeta K}\left[\left(g_{t t}-\varpi \Omega g_{\varphi \varphi}\right) D_{3}-g_{\varphi \varphi}(\Omega-\varpi) D_{4}+\left\{A_{2} g_{\varphi \varphi}(\Omega-\varpi)-A_{1}\left(g_{t t}-\varpi \Omega g_{\varphi \varphi}\right)\right\} D_{5}\right]
$$

where

$$
\begin{aligned}
& D_{3}=€^{A B}\left[K R C g_{\varphi A} q_{B}-K \zeta g_{t A}\left(\bar{\Phi}_{, B}+\bar{j} \Omega_{, B}\right)\right] \\
& D_{4}=€^{A B}\left[K R C g_{t A} q_{B}-K \zeta g_{t A}\left(\bar{\Phi}_{, B}+\bar{j} \Omega_{, B}\right)\right], \\
& D_{5}=€^{A B} g_{\varphi A} g_{t B} .
\end{aligned}
$$

It is evident from $(3.10)$ and $(3.11 a, b)$ that the frame dragging effect as well as the gradient of $\Omega$ comprise an additional rotation $\overline{\bar{\Omega}}$ of the matter part of fluid arising due to its merdional circulation that couples to the heat flow and the gradient of the injection energy.

From (2.13) with the aid of the first relation of (3.8), we get

$q_{A}=\frac{1}{R C}\left[\left(\bar{\Phi}_{, A}+\bar{j} \Omega_{, A}\right)+\frac{A_{1}}{K} g_{t A}-\frac{A_{2}}{K} g_{\varphi A}+\frac{I \sqrt{-g}}{K} €_{A B} w^{B}\right]$

From (2.15) and the third relation of (2.16a), we obtain

$$
V^{A}=\frac{\left(u_{t}\right)^{2}}{\zeta \sqrt{-g}} €^{A B}\left(\bar{j}_{, B}-l \bar{\varepsilon}_{, B}\right) .
$$

It follows from (3.12) and (3.13), on account of (3.2), that

$\bar{\Omega}_{, A} V^{A}=\overline{\bar{\Omega}}_{, A} V^{A}+\frac{1}{\bar{j}}\left(R C q_{A}-\bar{\Phi}_{, A}\right) V^{A}+\frac{1}{K}\left\{\left(A_{2} g_{\varphi A}-A_{1} g_{t A}\right) V^{A}-\bar{I} \sqrt{-g} €_{A B} V^{A} w^{B}\right\}$

The term on the left hand side of (3.14) describes the differential rotation of the matter part of fluid along the poloidal component of thermal-fluid vorticity vector and the first term on right hand side represents differential rotation due to meridional circulation of the matter part of fluid along the poloidal component of the thermal - fluid vorticity vector, the second term is the action of difference of poloidal component of heat flow and the gradient of injection energy along the poloidal component of thermal fluid vorticity, and the last term shows the contribution of non-diagonal metric tensor components and the cross-product of poloidal thermal-fluid vorticity and the meridional velocity of matter part of fluid. If we assume that the space-time is circular, (3.14) takes the form

$$
\Omega_{, A} V^{A}=\frac{R C}{\bar{j}} q_{A} V^{A}-\bar{I} \sqrt{-g} €_{A B} V^{A} w^{B}-\frac{1}{\bar{j}} \bar{\Phi}_{, A} V^{A},
$$

which exhibits that the differential rotation of matter part of fluid along the thermal-fluid vorticity vector causes 
i. the twist of the matter part of fluid's vortex lines ${ }^{10}$ due to first term on the left hand side of (3.15),

ii. thermal-fluid vorticity vector is non-parallel to the matter part of fluid's meridional velocity due to the second term and

iii. the injection energy per particle varies along the thermal-fluid vorticity vector due to third term.

This simple interpretation of (3.15) when extended to (3.14) in the case of non-circular spactime indicates the complication of the effect of differential rotation along the thermal-fluid vorticity.

The above result in turn suggests that the thermal-fluid vorticity may have a strong bearing on differential rotation of matter part of fluid because of the interdependence of heat flow and fluid's vorticity. We now investigate the impact of differentia rotation of matter part of fluid in a frame of reference in which thermal -fluid helicity is conserved in the following section.

\section{Differential rotation of thermal-fluid vorticity flux surfaces}

In a Carter's model of a heat conducting fluid, ${ }^{16,17}$ it is observed that the non-conservation of thermal-fluid helicity is a generic property in a matter part of fluid's frame $u^{a}{ }^{10}$ However, a frame of reference with 4-velocity $U^{a}$ may be constructed in which the thermal -fluid helicity is conserved. ${ }^{10}$ The relation between the thermal -fluid helicity conserving 4-velocity $U^{a}$ and the matter part of fluid's 4-velocity may be expressed as: ${ }^{10}$

$$
U^{a}=\Gamma\left(u^{a}+\bar{v}^{a}\right)
$$

Where $\Gamma=\left(1-\bar{v}^{2}\right)^{-\frac{1}{2}} \quad$ and $\quad u_{a} \bar{v}^{a}=0 \quad$ and $U^{a} U_{a}=-1$. The 4-velocity $U^{a}$ will be the thermal-fluid helicity conserving if it satisfies the following condition

$$
£_{U} W_{a b}=0 \text {, }
$$

where $£_{U}$ represent the Lie derivative along the vector $U^{a}$. Since $W_{a b}$ is a curl, it follows from (4.2) that

$$
W_{a b} U^{b}=0 \text {, }
$$

The velocity of deviation from the matter part of fluid's 4-velocity $u^{a}$ is denoted by $\bar{v}^{a}$ which is given by ${ }^{10}$.

$$
\bar{v}^{a}=\frac{1}{V^{2}} \eta^{a b c d} \mathrm{E}_{b} V_{c} u_{d}
$$

This gives the transport velocity of thermal-fluid vortex lines generated by $V^{a}$. The magnetic part of $W_{a b}$ is defined by

$$
\varsigma^{a}={ }^{*} W^{a b} U_{b},
$$

It is found that (see for details ${ }^{10}$ )

$$
\varsigma^{a}=\frac{1}{V} \sqrt{V^{2}-\mathrm{E}^{2}} \quad V^{a} \text {. }
$$

On account of (4.3) and (4.5), one may find that

$$
\begin{aligned}
& { }^{*} W^{a b}=U^{a} \varsigma^{b}-U^{b} \varsigma^{a} . \\
& W^{a b}=\eta^{a b c d} \varsigma_{c} U_{d} .
\end{aligned}
$$

Because of (4.7b) $\varsigma^{a}$ and $U^{a}$ are eigenvector of thermal -fluid vorticity 2-form $W_{a b}$ with zero eigenvalues. Hence, by Carter's argument, ${ }^{26}$ these two independent eigenvectors span a family of timelike 2-surfaces which have been referred to as thermal -fluid vorticity flux surfaces. ${ }^{10}$ The 4-velocity vector $U^{a}$ can now be interpreted as to represent the 4- velocity of those matter particles which are confined to the thermal -fluid vorticity flux surfaces. In fact, these time like surfaces are material surfaces which will be shown later in the text below. Consequently, the 4-velocity $U^{a}$ may be thought of as a 4-velocity of thermal-fluid flux surfaces.

It follows from (2.1a), (4.1), and (4.3) that

$$
\mathrm{E}_{a}=-W_{a b} \bar{v}^{b},
$$

which because of (2.9) and (2.5a.b) takes the form

$$
\mathrm{E}_{a}=\frac{1}{K}\left[-\bar{A}_{1} \xi_{(t)} a+\bar{A}_{2} \xi_{(\varphi) a}-\bar{I}_{a} \eta b c d \bar{v}^{b} \xi_{(t)}^{c} \xi_{(\varphi)}^{d}\right],
$$

where

$$
\begin{aligned}
& \bar{A}_{1}=\left(\bar{\varepsilon}_{, a} \bar{v}^{a}\right) g_{\varphi \varphi}+\left(\bar{j}_{, a} \bar{v}^{a}\right) g_{t \varphi}, \\
& \bar{A}_{2}=\left(\bar{\varepsilon}_{, a} \bar{v}^{a}\right) g_{t \varphi}+\left(\bar{j}_{, a} \bar{v}^{a}\right) g_{t t} .
\end{aligned}
$$

The toroidal components of $E_{a}$ are obtainable from (4.9) and $(4.10 \mathrm{a}, \mathrm{b})$ in the following form:

$$
\mathrm{E}_{t}=\left(\bar{\varepsilon}_{, a} \bar{v}^{a}\right), \mathrm{E}_{\varphi}=\left(\bar{j}_{, a} \bar{v}^{a}\right) \text {. }
$$

From (2.6) and (4.1), we get

$$
U^{a}=\Gamma\left\{\zeta\left(\xi_{(t)}^{a}+\Omega \xi_{(\varphi)}^{a}\right)+\overline{\bar{v}}^{a}\right\},
$$

where

$$
\overline{\bar{v}}^{a}=w^{a}+\bar{v}^{a} .
$$

The angular velocity corresponding to (4.12) can be defined as

$$
\hat{\Omega}=\frac{U^{\varphi}}{U^{t}} \approx \Omega+\breve{\Omega},
$$

where the second and higher order terms in the expansion are ignored and first order term is retained as

$$
\breve{\Omega}=\frac{1}{\Gamma \zeta}\left(\overline{\bar{v}}^{\varphi}-\Omega \overline{\bar{v}}^{t}\right) .
$$

Using (4.14) in (4.15) and taking (3.3) into account, we find that

$$
\breve{\Omega}=\frac{1}{\Gamma}+\frac{1}{\Gamma \zeta}\left(\bar{v}^{\varphi}-\Omega \bar{v}^{t}\right) .
$$

From (4.9), we have $\mathrm{E}_{a} \bar{v}^{a}=0$ which because of (4.9) gives

$$
\mathrm{E}_{t} \bar{v}^{t}+\mathrm{E}_{\varphi} \bar{v}^{\varphi}=0
$$

If it obeys the condition that

$$
\bar{v}^{t}=\left(\frac{\bar{j}, a \bar{v}^{a}}{\bar{\varepsilon}_{, a} \bar{v}^{a}}\right) \bar{v}^{\varphi} .
$$

Substitution for $\bar{v}^{\varphi}$ from (4.17) in the second term on right hand side of (4.16) gives

$$
\breve{\Omega}=\frac{1}{\Gamma} \overline{\bar{\Omega}}-\frac{\bar{v}^{t}}{\Gamma_{\zeta} \mathrm{E}_{\varphi}}\left(\mathrm{E}_{t}+\Omega \mathrm{E}_{\varphi}\right)
$$


which, on account of (4.14), yields that

$$
\hat{\Omega}=\Omega+\frac{1}{\Gamma}-\frac{\bar{v}^{t}}{\Gamma_{\zeta} \mathrm{E}_{\varphi}}\left(\mathrm{E}_{t}+\Omega \mathrm{E}_{\varphi}\right)
$$

It is seen from (4.20) that the rotational velocity of those particles which are confined to thermal-fluid vorticity flux surfaces is composed of the rotational velocity of matter part of fluid and the toroidal components of heat flow vector. In other words, (4.20) represents the rotational velocity of thermal-fluid flux surfaces caused by the rotational velocity of the matter part of fluid and the heat flows.

In order to find the magnetic part $\varsigma^{a}$ of the fluid-thermal voracity 2 -form $W_{a b}$, we substitute (4.12) in the right hand side of (4.5) and simplify with the aid of $(2.5 \mathrm{a}, \mathrm{b}),(2.10),(2.16 \mathrm{a}, \mathrm{b})$ to get

$$
\varsigma^{a}=\Gamma \zeta\left[-\frac{\bar{I}}{\zeta} u_{\varphi} \xi_{(t)}^{a}+\frac{\bar{I}}{\zeta} u_{t} \xi_{(\varphi)}^{a}-\eta^{a b c d} R_{b} \xi_{(t) c} \xi_{(\varphi) d}-\eta^{a b c d} \overline{\bar{v}}_{b} Q_{c} \xi_{(t) d}+\eta^{a b c d} \overline{\bar{v}}_{b} \bar{Q}_{c} \xi_{(\varphi) d}\right] .
$$

From now on, we assume that

$$
\varsigma^{t}=o \text { and } \varsigma^{\varphi}=o \text {. }
$$

It is to be noted that because of (4.22) the covariant toroidal components $s_{t}$ and $\varsigma_{\varphi}$ are non-zero due to non-circularity of spacetime and the presence of meridional circulation of matter part of fluid. This in turn implies that $\bar{I}$ is not zero because $\bar{I}=U_{t} \varsigma_{\varphi}-U_{\varphi} \varsigma_{t}$ cannot vanish. Making use of (4.22) with the aid of (4.21), we find that

$$
l \varpi=1+\frac{\zeta K}{u_{t} g_{\varphi \varphi} \sqrt{-g}}\left[€^{A B} \overline{\bar{v}}_{A} Q_{B}-€^{A B} R_{A} g_{\varphi B}\right],
$$

which describes the frame dragging effect in terms of the meridional circulation of the matter part of fluid coupled to the transport velocity of thermal -fluid vortex lines and the gradients of effective energy and angular momentum per particle corresponding to the matter part of fluid.

Since $W_{a b}$ is a curl, we have

$$
{ }^{*} W_{; b}^{a b}=0 \text {. }
$$

Substitution of (4.7a) into (4.24) gives that

$$
U_{, b}^{a} \varsigma^{b}-\varsigma_{, b}^{a} U^{b}+U^{a} \varsigma_{; b}^{b}=U_{; b}^{b} \varsigma^{a} .
$$

By virtue of our assumption (4.22), we find from (4.25) for $a=t, \varphi$ that

$$
\begin{aligned}
& U_{, b}^{t} \varsigma^{b}+U^{t} \varsigma_{; b}^{b}=0, \\
& U_{, b}^{\varphi} \varsigma^{b}+U^{\varphi} \varsigma_{; b}^{b}=0 .
\end{aligned}
$$

Making use of (4.14) in (4.26b), one may obtain

$$
\hat{\Omega}_{, b} \varsigma^{b}+\hat{\Omega} U_{, b}^{t} \varsigma^{b}+\hat{\Omega} U^{t} \varsigma_{; b}^{b}=0 .
$$

Multiplying (4.26a) by $\hat{\Omega}$ and subtracting the resulting equation from (4.27), we get

$$
\hat{\Omega}_{, A} \varsigma^{A}=0,
$$

which bears a striking resemblance with Ferraro's law of isorotation in RMHD. ${ }^{19}$ This result states that the differential rotation of thermal -fluid vorticity flux surfaces is constant along the magnetic part of thermal-fluid vorticity 2-form that represents the thermal- fluid vorticity given by (4.6). It follows from (2.3), (4.6) and (4.28) that

$$
{ }^{*} \mu \hat{\Omega}_{, A} \omega^{A}=-\alpha q \hat{\Omega}_{, A} \hat{\omega}^{A},
$$

which can be interpreted as follows. The left hand side of (4.29) is the gravitational isorotation in the sense of Glass ${ }^{9}$ and the right hand side is the thermal isorotation by virtue of $\hat{\omega}^{A}$ that represents the poloidal component of vorticity of heat flow lines. Thus (4.29) says that the gravitational isorotation of thermal -fluid vorticity flux surfaces is balanced by counter rotating thermal isorotation. The coupled effects of differential rotation of matter part of fluid with that of thermal-fluid vorticity flux surfaces can be seen by substituting (4.20) in (4.29).

\section{It follows from (4.25) that}

$$
£_{U} \varsigma^{a}=U^{a} \varsigma_{; b}^{b}-\varsigma^{a} U_{; b}^{b},
$$

which shows that the Lie derivative of $\varsigma^{a}$ is a linear combination of $U^{a}$ and $\varsigma^{a}$. Consequently, $\varsigma^{a}$ obeys Greenberg's transport law ${ }^{25}$ and hence because of (4.6) thermal-fluid vortex lines are material lines. ${ }^{6}$ This in turn implies that a family of timelike 2 -suraces spanned by $U^{a}$ and $\varsigma^{a}$ (or equivalently $V^{a}$ ) is a family of material thermalfluid vortex surfaces. The differential rotation of thermal-fluid vortex surfaces given by (4.29) induces differential rotation in the matter part of fluid and vice-versa.

\section{Conservation of energy and angular momentum currents}

In this section we discuss the symmetry related conservation laws of energy and angular momentum currents associated with a system composed of a heat conducting fluid. As is known ${ }^{21}$ that the energy and angular momentum currents associated with the system under spacetime symmetry assumption corresponding to the time like Killing vector $\xi_{(t)}^{a}$ and the spacelike Killing vector $\xi_{(\varphi)}^{a}$ are, respectively, defined by

$$
\chi_{(t)}^{a}=T_{b}^{a} \xi_{(t)}^{b},
$$

$$
\chi_{(\varphi)}^{a}=T_{b}^{a} \xi_{(\varphi)}^{b},
$$

Where $\chi_{(t)}^{a}$ and $\chi_{(\varphi)}^{a}$ denote, respectively, the energy and angular momentum currents. $T_{b}^{a}$ is the energy-momentum tensor of a heat conducting fluid given by

$$
T_{b}^{a}=\left(n^{*} \mu+{ }^{*} s^{*} \theta\right) u^{a} u_{b}+\psi \delta_{b}^{a}+u^{a} q_{b}+q^{a} u_{b}+\frac{\beta}{{ }^{*} \theta} q^{a} q_{b} .
$$

Since $\xi_{(t)}^{b}$ is a Killing vector, it follows from (5.1) that

$$
\chi_{(t) ; a}^{a}=0
$$

In consequence of the conservation law $T_{b ; a}^{a}=0$. Substituting (5.2) in (5.1a) and using the resulting expression in (5.3), we find that

$$
n\left[\left({ }^{*} \mu+\frac{{ }^{*} s^{*} \theta}{n}\right) u_{t}+\frac{q_{t}}{n}\right]_{, a} u^{a}+\left[\left(u_{t}+\frac{\beta}{{ }^{*} \theta} q_{t}\right) q^{a}\right]_{; a}=0,
$$


In consequence of baryon conservation law $\left(n u^{a}\right)_{; a}=0$. Eq. (5.4) gives the conservation of energy current associated with the motion of a heat conducting fluid. We now introduce the energy per baryon defined by

$$
-E=\left({ }^{*} \mu+\frac{{ }^{*} s^{*} \theta}{n}\right) u_{t}+\frac{q_{t}}{n} .
$$

From (5.4) and (5.5), on account of the first relation of (2.21), we find that

$$
n E_{, a} u^{a}+\left(\frac{\varepsilon}{{ }^{*} \theta} q^{a}\right) ; a=0,
$$

which shows that there is a mutual exchange between the energy per baryon of the matter part of fluid and the flux of effective thermal energy per entropon per unit of local temperature of the entropy fluid coupled to heat flow vector. Similarly, we find from (5.1b) and (5.2) by invoking the conservation law of energy-momentum tensor that

$$
n\left[\left({ }^{*} \mu+\frac{{ }^{*} s^{*} \theta}{n}\right) u_{\varphi}+\frac{q_{\varphi}}{n}\right], u^{a}+\left[\left(u_{\varphi}+\frac{\beta}{{ }^{*} \theta} q_{\varphi}\right) q^{a}\right]: a=0 .
$$

The angular momentum per baryon is defined as

$$
L=\left[\left({ }^{*} \mu+\frac{{ }^{*} s^{*} \theta}{n}\right) u_{\varphi}+\frac{q_{\varphi}}{n}\right] .
$$

It follows from (5.7) and (5.8), in view of the second relation of (2.21), that

$$
n L_{, a} u^{a}+\left(\frac{j}{{ }^{*} \theta} q^{a}\right)_{; a}=0
$$

which exhibits the mutual exchange between the matter part of fluid's angular momentum per baryon and the flux of angular momentum per entropon per unit of local temperature of the entropy fluid coupled to heat flow vector. Thus (5.6) and (5.9) describe the dynamic interaction between the matter part of fluid and the entropy fluid during the course of evolution via the mutual exchange of fluid's both energy and angular momentum per baryon with that of the flux of both the effective energy and effective angular momentum per entropon per unit of local temperature coupled to heat flow vector generated by the entropy fluid. It is worthwhile to notice that both (5.6) and (5.9) differ from the usual Bernoulli's theorem ${ }^{30}$ in the sense that the energy and angular momentum per baryon of the matter part of fluid is not constant along the matter part of fluid's flow lines. Eq. (5.6) and (5.9) represent the mutual exchange between the energy and angular momentum per baryon of mater part of fluid and flux of both the effective energy and effective angular momentum per entropon per unit of local temperature coupled to heat flow vector created by the entropy fluid in response to dynamic interaction with the motion of the matter part of fluid. This result looks simple but vindicates the hypothesis that the entropy element can be thought of as a mass less fluid constituted by entropon. It is expected that further investigation will reveal the elegance of the entropy fluid in the dynamic interaction with the motion of a heat conducting matter in Carter's model. ${ }^{16,17}$

\section{Conclusion}

The present work is focused on the study of dynamic interaction of the entropy fluid with the motion of matter part of fluid based on Carter's two-fluid model under the assumption that the space-time representing the gravitational field of a self-gravitating heat conducting fluid is stationary axisymmetric and non-circular. It is found that the rotational velocity of the matter part of fluid is modified due to noncircularity assumption of space-time which ensures the existence of its meridional circulation that induces an additional rotational effect. The effective energy and the effective angular momentum per particle of both the fluids contribute to the generation of rotational velocity. The differential rotation of the matter part of fluid along the thermal-fluid vector generates twist of the matter part of fluid's vortex lines besides other effects. Ferraro's like law of isorotation is obtained in the case of differentially rotating thermal-fluid vorticity flux surfaces in a frame of reference in which thermal-fluid helicity is conserved. Finally, by deriving the conservation laws of energy and angular momentum currents associated with the system of heat conducting fluid, it is demonstrated that there is a mutual exchange between both the energy and angular momentum per baryon of the matter part of fluid and of flux of both effective energy and effective angular momentum per entropon per unit of local temperature coupled to the heat flow vector created by the entropy fluid.

The above results indicate that the novel idea of the entropy element as a fluid will provide a new insight into the physical processes associated with the evolution of hot matter that departs from thermodynamic equilibrium. The notion of thermal equilibrium defined by the vanishing of heat flow vector seems to be a mathematical artifact because there is no thermodynamic condition that states the physical condition for the vanishing of heat flow vector in bringing the system to a thermal equilibrium except to assuming the vanishing of heat flow vector in addition to Born rigidity condition of fluid's flow lines. If it is true, then the question is how the contribution of heat coupled to the entropy entrainment is eliminated from the EOS of a heat conducting fluid. This question requires answer.

\section{Acknowledgements}

None

\section{Conflict of interest}

Author declares that there is no conflict of interest.

\section{References}

1. C Constantinou, B Mucciali, M Prakash, et al. Thermal properties of hot and dense matter with finite range interactions. Phys Rev C. 2015;92:025801.

2. JL Friedman, N Stergioulas. Rotating Relativistic Stars. Cambridge Monographs on Mathematical Physics, UK:Cambridge University press; 2013. p. 429.

3. L Lindblom. On the Analyticity of Certain Stationary Nonvacuum Einstein Spacetimes. Astroph J. 1976;208:873-880.

4. M Born. Die träge Masse und das Relativitätsprinzip. Annlen Phys. 1909;333(3):571.

5. C Eckart. The Thermodynamics of Irreversible Processes. III. Relativistic Theory of the Simple Fluid. Phys. Rev. 1940;58(10):919-924.

6. M Tsamparlis, DP Masson. On spacelike congruences in general relativity. J Math Phys. 1983;24:1577.

7. JN Cook, SL Shapiro, BC Stephens. Magnetic Braking and Viscous Damping of Differential Rotation in Cylindrical Stars. Astroph J. 2003;599(2):1272.

8. RH Boyer. Rotating fluid masses in general relativity. II. Proc Camb Phil Soc. $1966 ; 62(3): 495-501$. 
9. EN Glass. Rotating steady state configurations in general relativity. J Math Phys. 1977;18:708

10. G Prasad. Vorticity and helicity in relativistic heat-conducting fluid. Class Quant Grav. 2017;34:245011.

11. D Bekenstein. Helicity conservation laws for fluids and plasmas. Astroph J. 1987;319:207-214.

12. F Weber, R Negreiros. Phase Transitions in Dense Baryonic Matter and Cooling of Rotating Neutron Stars. Acta Physica Pol. 2010;30:701-710.

13. R Negreiros, S Schramn. Thermal evolution of neutron stars in two dimensions. Phys Rev D. 2012;85:104019.

14. AY Potekhin, A De Lucas, JA Pons. Neutron Stars-Cooling and Transport. Space Sci Rev. 2015;191(1-4):239-291.

15. SK Lander, $\mathrm{N}$ Andersson. Heat Conduction in Rotating Stars. 2018;479(3):4207-4215.

16. B Carter. Relativistic Fluid Dynamics in Lecture Notes in Mathematics In: Angelo M Anile, Yvonne Choquet Bruhat, editors. Lecture Notes in Mathematics. Volume 1385, Berlin, Heidelberg: Springer;1989. p.1-64

17. CS Lopez Monsalov, N Andersson. Thermal dynamics in general relativity. Proc Roy Soc London A. 2011;467(2127):738-759.

18. W Israel, JM Stewart. Transient relativistic thermodynamics and kinetic theory. Ann Phys. 1979;118(2):341-372.

19. D Priou. Comparison between variational and traditional approaches to relativistic thermodynamics of dissipative fluids. Phys Rev D 1991;43(4):1223.

20. G Prasad. Thermal Vorticity and Thermal Chirality in Relativistic Thermally Conducting Fluids. Class Quant Grav. 2018.
21. B Carter. Black Hole Equilibrium States. In: Black Hole. Les Houches, $\mathrm{C}$ Dewitt, editors. New York. 1973. p. 61-214.

22. E Gourgoulhon, C Marlakis, K Uryu, et al. Magnetohydrodynamics in stationary and axisymmetric spacetimes: A fully covariant approach. Phys Rev D. 2011;83:104007.

23. A Lichnerowicz. Relativistic Hydrodynamics and Magnetohydrodynamics. New York: Benjamin. 1967.

24. J Ehlers. Contributions to the relativistic mechanics of continuous media. Gen Rel Grav. 1993;25:1225-1266.

25. PJ Greenberg. The general theory of space like congruences with an application to vorticity in relativistic hydrodynamics. J Math Anal Appl. 1970;30(1):128-143.

26. B Carter. Perfect Fluid and Magnetic Field Conservation Laws in the Theory of Black Hole accretion Rings. In: Active Galactic Nuclei. C Hazard, S Mitton, editors. UK: Cambridge University press; 1979. p.273-279

27. G Prasad. Fluid-magnetic helicity in axisymmetric stationary relativistic magnetohydrodynamics. General Relativity and Gravitation. 2017;49:126.

28. JM Bardeen. Rotating Stars and Disk in Black Hole. In: Les Houches, C Dewitt, BS Dewitt, editors. New York: Gordon Breach; 1973. p 241-290.

29. AK Raychaudhuri. Ferraro's theorem in non-flat space time. Mont Not Roy Astron Soc. 1979;189:39-43.

30. JD Bekennstein, E Oron. New conservation laws in general-relativistic magnetohydrodynamics. Phys Rev D. 1978;18:1809. 